\title{
Interplay between $s-d$ exchange interaction and Rashba effect: Spin-polarized transport
}

\author{
W. Yang, Kai Chang, ${ }^{\text {a) }}$ X. G. Wu, and H. Z. Zheng \\ SKLSM, Institute of Semiconductors, Chinese Academy of Sciences, P.O. Box 912, 100083 Beijing, China
}

F. M. Peeters

Department of Physics, University of Antwerp, Campus Drie Eiken, B-2610 Antwerp, Belgium

(Received 23 February 2006; accepted 17 August 2006; published online 26 September 2006)

\begin{abstract}
The authors investigate the spin-polarized transport properties of a two-dimensional electron gas in a $n$-type diluted magnetic narrow gap semiconductor quantum well subjected to perpendicular magnetic and electric fields. Interesting beating patterns in the magnetoresistance are found which can be tuned significantly by varying the electric field. A resonant enhancement of spin-polarized current is found which is induced by the competition between the $s$ - $d$ exchange interaction and the Rashba effect [Y. A. Bychkov and E. I. Rashba, J. Phys. C 17, 6039 (1984)]. (c) 2006 American Institute of Physics. [DOI: 10.1063/1.2357888]
\end{abstract}

Recently, spin related phenomena in semiconductors have acquired a renewed interest because of the possibility of using the spin degree of freedom of carriers to construct spintronic devices. ${ }^{1}$ One of the central issues in semiconductor spintronics is the creation of spin-polarized current. In a diluted magnetic semiconductor (DMS), the large $s-d$ exchange interaction between the conduction electrons and the localized magnetic ions provides the possibility of tailoring the electron spin splitting and consequently modifies significantly the transport property of spin-polarized electrons. Via vertical tunneling through DMS and ferromagnetic metallic junctions, efficient spin injection into semiconductors has been demonstrated. ${ }^{2-7}$ The motion of an electron spin in a DMS or conventional semiconductor may also be modified by the so-called Rashba spin-orbit interaction (SOI). ${ }^{8}$ This interaction offers the opportunity to manipulate electron spin in a two-dimensional electron gas (2DEG) via an external electric field instead of a magnetic field. ${ }^{9}$ The coupling may lead to interesting effects, e.g., the recently demonstrated ${ }^{10,11}$ spin Hall effect. ${ }^{12}$ Experimentally, it was found that the Rashba SOI can modify significantly the magnetotransport property of 2DEG in an InGaAs/InAlAs heterostructure. ${ }^{13}$

In this letter, we investigate theoretically the spin transport properties of a $2 \mathrm{DEG}$ in a paramagnetic DMS in the presence of perpendicular magnetic and electric fields. We include both the $s$ - $d$ exchange interaction, which can be tuned by the magnetic field, and the Rashba SOI, whose strength can be modified by a perpendicular electric field. We found that a strong resonance-enhanced spin-polarized current may appear at a critical Rashba SOI strength even at low magnetic fields.

Let us consider a 2DEG confined in a Mn-based DMS quantum well in the presence of perpendicular magnetic and electric fields. The Hamiltonian of the conduction electron including the SOI and the $s-d$ exchange interaction ${ }^{14}$ is given by

\footnotetext{
a) Author to whom correspondence should be addressed; electronic mail:
} kchang@red.semi.ac.cn

$$
\hat{H}=(\mathbf{p}+e \mathbf{A})^{2} / 2 m_{e}+\alpha / \hbar\left[(\mathbf{p}+e \mathbf{A}) \times \mathbf{e}_{z}\right] \cdot \boldsymbol{\sigma}+\hat{H}_{z}+\hat{H}_{\mathrm{ex}},
$$

where $\alpha$ is the Rashba SOI strength, $\hat{H}_{z}=g_{e} \mu_{B} B \hat{\sigma}_{z} / 2$ is the intrinsic Zeeman term, and $\hat{H}_{\mathrm{ex}}=-\Sigma_{j} J\left(\mathbf{r}-\mathbf{R}_{j}\right) \boldsymbol{\sigma} / 2 \cdot \mathbf{S}_{j}$ is the $s$ - $d$ exchange interaction between the conduction electron $\operatorname{spin}(\boldsymbol{\sigma} / 2)$ and the localized spins $\left(\mathbf{S}_{j}\right)$. Within the meanfield approximation, $\hat{H}_{\mathrm{ex}}=-N_{0} \alpha_{\mathrm{ex}} x\left\langle S_{z}\right\rangle \hat{\sigma}_{z} / 2$, where $N_{0} \alpha_{\mathrm{ex}}$ describes the strength of the $s$ - $d$ exchange interaction, $x$ is the fractional occupancy of the Mn ions on cation sites, and $\left\langle S_{z}\right\rangle$ is the thermal average of the Mn spin.

The eigenvalues and eigenstates of the electron are $(n=1,2, \ldots)$

$$
\begin{aligned}
& E_{n}^{( \pm)}=(n \pm \delta) \hbar \omega_{c}, \\
& E_{0}=\left(\hbar \omega_{c}-\Delta_{Z}\right) / 2, \\
& \delta=\sqrt{\left[\Delta_{Z} /\left(\hbar \omega_{c}\right)-1\right]^{2} / 4+2 n\left[\alpha /\left(l_{B} \hbar \omega_{c}\right)\right]^{2}}, \\
& \psi_{n, k_{y}}^{( \pm)}=e^{i k_{y} y} / \sqrt{L_{y}}\left[C_{n}^{( \pm)} \phi_{n-1}|\uparrow\rangle+D_{n}^{( \pm)} \phi_{n}|\downarrow\rangle\right], \\
& \psi_{0, k_{y}}=e^{i k_{y} y} / \sqrt{L_{y}} \phi_{0}|\downarrow\rangle .
\end{aligned}
$$

Here $\Delta_{Z}=g_{e} \mu_{B} B-N_{0} \alpha_{\text {ex }} x\left\langle S_{z}\right\rangle, L_{y}$ is the sample length along the $y$ axis, $\left\{\phi_{n}\right\}$ are harmonic oscillator functions centered at $x_{0}=-k_{y} l_{B}^{2}\left[l_{B}=\sqrt{\hbar /(e B)}\right], \quad C_{n}^{(+)}=-D_{n}^{(-)}=P_{n} / A_{n}, \quad C_{n}^{(-)}=D_{n}^{(+)}$ $=1 / A_{n}$ for $\Delta_{Z}<\hbar \omega_{c}, C_{n}^{(+)}=D_{n}^{(-)}=1 / A_{n}, C_{n}^{(-)}=-D_{n}^{(+)}=-P_{n} / A_{n}$ for $\Delta_{Z}>\hbar \omega_{c}, \quad P_{n}=\sqrt{2 n l_{B}^{-1}} \alpha /\left(\left|\Delta_{Z}-\hbar \omega_{c}\right| / 2+\hbar \omega_{c} \delta\right)$, and $A_{n}$ $=\sqrt{1+P_{n}^{2}}$. It is interesting to notice that the zeroth Landau level (LL) $\left(\psi_{0, k_{y}}\right)$ is decoupled from other LLs and is always a spin-down eigenstate when the strength of the Rashba SOI $(\alpha)$ varies.

The broadening of the LLs induced by impurity scattering is obtained from the Fermi golden rule $\Gamma_{n k_{y} \lambda}$ $=\hbar \sum_{n^{\prime} k_{y}^{\prime} \lambda^{\prime}} W_{n k_{y} \lambda, n^{\prime} k_{y}^{\prime} \lambda^{\prime}}\left(\lambda, \lambda^{\prime}= \pm\right)$, where $W_{i, j}$ is the impurity scattering rate from initial state $|i\rangle$ to final state $|j\rangle$. Based on the quantum Boltzmann equation, ${ }^{15}$ the conductivity is given by 


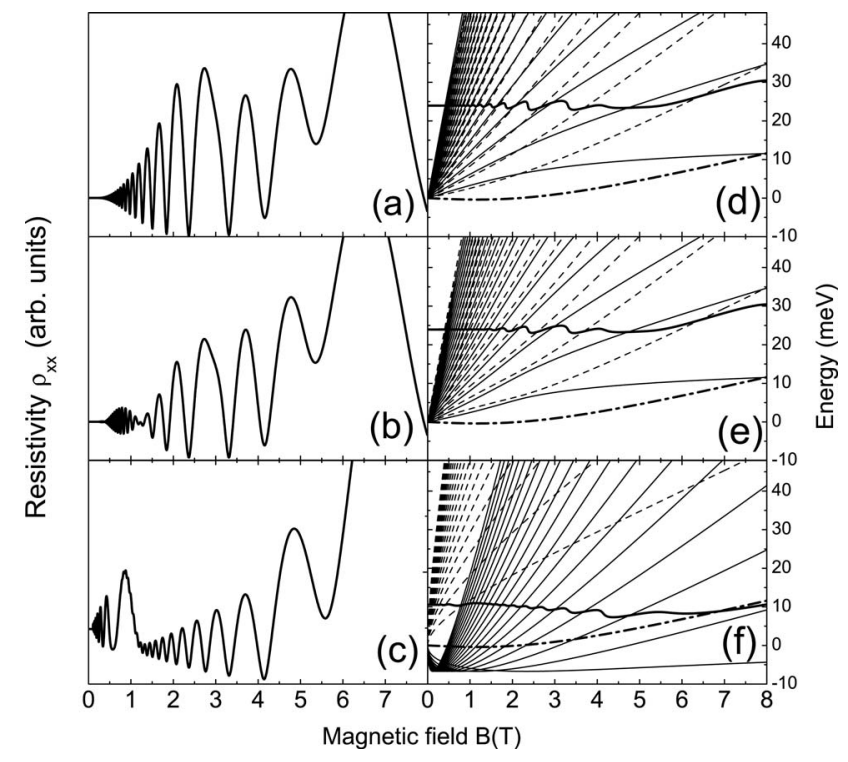

FIG. 1. [(a)-(c)] Longitudinal magnetoresistivity $\rho_{x x}$ and [(d)-(f)] energy spectra at $T=1 \mathrm{~K}$ as a function of magnetic field for $\alpha=0,5$, and $160 \mathrm{meV} \mathrm{nm}$. The thickest solid lines in (d)-(f) denote the Fermi energies, while the thin solid and dashed lines denote the $(-)$ and $(+)$ branches of LLs, respectively. The thicker dash-dotted lines denote the zeroth spin-down LL.

$$
\sigma_{x x}=\frac{e^{2}}{2 k_{B} T \Re} \sum_{i, j} f\left(E_{i}\right)\left[1-f\left(E_{j}\right)\right] W_{i j}\left(\langle x\rangle_{i}-\langle x\rangle_{j}\right)^{2},
$$

where $f\left(E_{i}\right)$ is the Fermi distribution function and $\langle x\rangle_{i}$ is the cyclotron center of state $|i\rangle$. The resistivity is obtained from $\rho_{x x}=\sigma_{x x} / S$, where $S=\left(\sigma_{x x}\right)^{2}+\left(\sigma_{x y}\right)^{2} \approx\left(e n_{e} / B\right)^{2}$.

In the presence of the SOI, the eigenstates of the electron are no longer pure spin-up or spin-down states, but a mixture of them (except $\psi_{0, k_{y}}$ ). The most widely used definition of the current polarization is $P_{J}=\left(\sigma_{x x}^{\uparrow}-\sigma_{x x}^{\downarrow}\right) /\left(\sigma_{x x}^{\uparrow}+\sigma_{x x}^{\downarrow}\right),{ }^{16}$ with

$$
\sigma_{x x}^{\uparrow, \downarrow}=\eta \sum_{i, j} f\left(E_{i}\right)\left[1-f\left(E_{j}\right)\right] W_{i j}\left(\langle x\rangle_{i}-\langle x\rangle_{j}\right)^{2} P_{j}^{\uparrow, \downarrow}
$$

where $\eta=e^{2} /\left(2 k_{B} T \Re\right) \quad$ and $\quad P_{j}^{\uparrow}=\left|C_{j}^{ \pm}\right|^{2}\left(P_{j}^{\downarrow}=\left|D_{j}^{ \pm}\right|^{2}\right) \quad$ is the spin-up (spin-down) probability of the final state $|j\rangle$. The definition of the current polarization $P_{J}$ is consistent with the usual definition in the case of zero SOI $(\alpha=0)$ and approaches zero in the case of strong SOI $(\alpha \rightarrow \infty)$ as expected. In the case of $\alpha=0$, our theoretical model can produce excellent agreement with recent experiment, demonstrating the validity of our theory. ${ }^{17}$

In order to investigate the interference between the $s-d$ exchange interaction and the Rashba effect, we consider a narrow band gap DMS 2DEG in $\mathrm{Hg}_{0.88} \mathrm{Mn}_{0.12} \mathrm{Te}$, where the Rashba SOI strength could be strong. ${ }^{18}$ The following parameters are used: $E_{g}=0.2 \mathrm{eV}, N_{0} \alpha_{\mathrm{ex}}=400 \mathrm{meV}, g_{e}=-41$, $x_{\text {eff }}=0.02, m_{e}=0.04 m_{0}$ ( $m_{0}$ is the free electron mass), $n_{I}=3$ $\times 10^{9} \mathrm{~cm}^{-2}$, and electron density $n_{e}=4 \times 10^{11} \mathrm{~cm}^{-2}$. ${ }^{14,19}$ The magnetoresistance $\rho_{x x}$ is shown in Figs. 1(a)-1(c) for different perpendicular electric fields, i.e., different Rashba SOI strengths $(\alpha=0,5$, and $160 \mathrm{meV} \mathrm{nm})$. The beating patterns, which are imposed on the Shubnikov-de Haas oscillations of $\rho_{x x}$, arise from the sweep of the $(-)$ branch LLs over the $(+)$ branch LLs. The behavior of $\rho_{x x}$ is changed significantly by introducing the Rashba SOI [see Figs. 1(e) and 1(f)], since the interplay between the $s-d$ exchange interaction and the

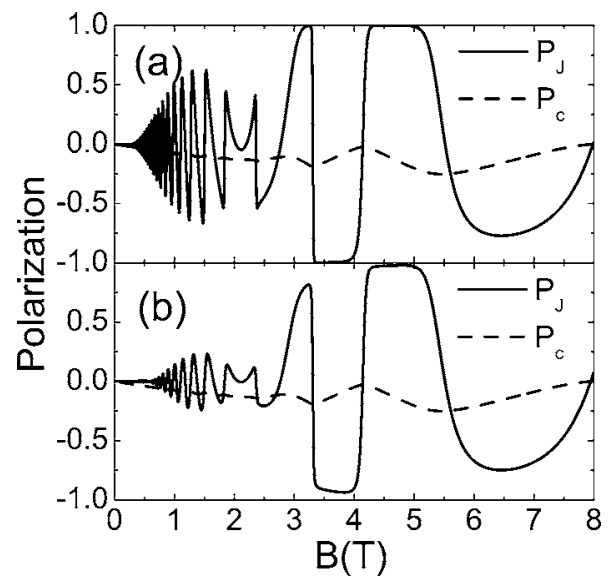

FIG. 2. Spin polarization of carrier $P_{c}$ and current $P_{J}$ for (a) $\alpha=0$ and (b) $\alpha=5 \mathrm{meV}$ nm at $T=1 \mathrm{~K}$.

Rashba SOI Ref. 20 changes the relative position of the two branches of LLs [see Eq. (2)]. For very strong Rashba SOI, $\rho_{x x}$ exhibits a very different behavior due to the separation of the two branches $( \pm)$ of LLs [see Figs. 1(c) and 1(f)].

In the absence of the Rashba SOI, a strong polarization of the current can be obtained by applying a strong magnetic field [see Fig. 2(a)]. It is interesting to find that the current polarization $P_{J}$ oscillates with increasing magnetic fields and approaches $\pm 100 \%$ at strong magnetic fields, while the carrier polarization $P_{c} \equiv\left(n_{\uparrow}-n_{\downarrow}\right) /\left(n_{\uparrow}+n_{\downarrow}\right)$ is always much smaller. The physical reason is that $P_{J}$ is related to the density-of-states polarization on the Fermi surface, while $P_{c}$ is determined by the carrier population. Thus our theoretical results show that a spin-polarized current in the DMS 2DEG can be realized by adjusting the magnetic field or electron density such that $E_{F}$ lies at the center of one spin-split LL.

The influence of Rashba SOI behaves like a momentumdependent in-plane effective magnetic field [see Eq. (1)]. It mixes the spin-up and the spin-down LLs except $\left(\psi_{0, k_{y}}\right)$ such that the electron eigenstates are no longer spin eigenstates, which, in most situations, reduces the spin polarization of the current [see Fig. 2(b)]. However, it is interesting to notice that the Rashba SOI could induce a resonant enhancement of the spin polarization of the current (see the deep dip at $\alpha_{m}$ in Fig. 3) for a fixed magnetic field. This interesting feature can be understood from the inset of Fig. 3. For strong Rashba SOI, the two branches $( \pm)$ of LLs consist of nearly equal

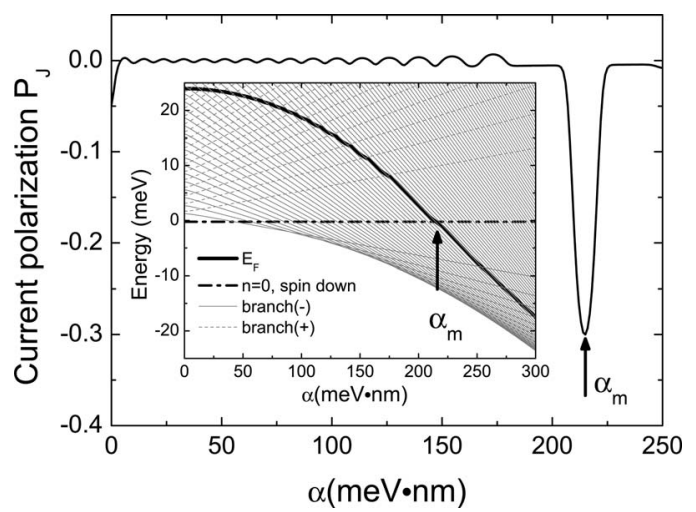

FIG. 3. Spin polarization of current vs the SOI strength for a fixed $B$ $=0.5 \mathrm{~T}$ at $T=1 \mathrm{~K}$. The inset shows the LLs and the Fermi energy as a function of the SOI strength. The line type is the same as in Fig. 1. $n_{e}=4$ $\times 10^{11} \mathrm{~cm}^{-2}$ and $x_{\text {eff }}=0.02$. 
spin-up and spin-down components, leading to a vanishingly small current polarization [see Eq. (6)] when they coincide with the Fermi energy. In contrast, the zeroth spin-down LL is a spin-down eigenstate and it contributes a pure spin-down current when it coincides with $E_{F}$. With increasing Rashba SOI, branch $(-)[(+)]$ LLs are shifted to lower (higher) energies, while the energy of the zeroth spin-down LL keeps constant [see Eq. (2)]. Consequently, the Fermi energy decreases and coincides with the zeroth spin-down LL, leading to resonant enhancement of the spin polarization of the current.

In conclusion, the competition between the $s$ - $d$ exchange interaction and the Rashba SOI provides us with an interesting possibility to create spin-polarized current in lowdimensional semiconductor structures. Strong spin-polarized current can be achieved even when the polarization of carriers is very small. A large spin polarization of current induced by the Rashba effect is predicted at low magnetic fields.

This work is partly supported by the NSF and MOST of China and the Bilateral Cooperation Programme between Flanders and China.

${ }^{1}$ G. A. Prinz, Science 282, 1660 (1998).

${ }^{2}$ S. A. Wolf, D. D. Awschalom, R. A. Buhrman, J. M. Daughton, S. von Molnár, M. L. Roukes, A. Y. Chtchelkanova, and D. M. Treger, Science 294, 1488 (2001).

${ }^{3}$ M. Oestreich, J. Hübner, D. Hägele, P. J. Klar, W. Heimbrodt, W. W. Rühle, D. E. Ashenford, and B. Lunn, Appl. Phys. Lett. 74, 1251 (1999).

${ }^{4}$ R. Fiederling, M. Keim, G. Reuscher, W. Ossau, G. Schmidt, A. Waag, and L. W. Molenkamp, Nature (London) 402, 787 (1999).

${ }^{5}$ Y. Ohno, D. K. Young, B. Beschoten, F. Matsukura, H. Ohno, and D. D. Awschalom, Nature (London) 402, 790 (1999).

${ }^{6}$ A. T. Hanbicki, B. T. Jonker, G. Itskos, G. Kioseoglou, and A. Petrou, Appl. Phys. Lett. 80, 1240 (2002).

${ }^{7}$ X. Jiang, R. Wang, R. M. Shelby, R. M. Macfarlane, S. R. Bank, J. S. Harris, and S. S. P. Parkin, Phys. Rev. Lett. 94, 056601 (2005); R. Wang, X. Jiang, R. M. Shelby, R. M. Macfarlane, S. S. P. Parkin, S. R. Bank, and J. S. Harris, Appl. Phys. Lett. 86, 052901 (2005).

${ }^{8}$ Y. A. Bychkov and E. I. Rashba, J. Phys. C 17, 6039 (1984).

${ }^{9}$ S. Datta and B. Das, Appl. Phys. Lett. 56, 665 (1990).

${ }^{10}$ Y. K. Kato, R. C. Myers, A. C. Gossard, and D. D. Awschalom, Science 306, 1910 (2004).

${ }^{11}$ J. Wunderlich, B. Kaestner, J. Sinova, and T. Jungwirth, Phys. Rev. Lett. 94, 047204 (2005).

${ }^{12}$ J. E. Hirsch, Phys. Rev. Lett. 83, 1834 (1999); S. Zhang, ibid. 85, 393 (2000); S. Murakami, N. Nagaosa, and S. C. Zhang, Science 301, 1348 (2003).

${ }^{13}$ J. Nitta, T. Akazaki, H. Takayanagi, and T. Enoki, Phys. Rev. Lett. 78, 1335 (1997).

${ }^{14}$ J. K. Furdyna, J. Vac. Sci. Technol. 21, 220 (1982); J. Appl. Phys. 64, R29 (1988).

${ }^{15}$ P. Vasilopoulos and F. M. Peeters, Phys. Rev. Lett. 63, 2120 (1989).

${ }^{16}$ S. I. Erlingsson, J. Schliemann, and D. Loss, Phys. Rev. B 71, 035319 (2005).

${ }^{17}$ F. J. Teran, M. Potemski, D. K. Maude, Z. Wilamowski, A. K. Hassan, D. Plantier, J. Jaroszynski, T. Wojtowicz, and G. Karczewski, Physica E (Amsterdam) 17, 335 (2003); W. Yang, Kai Chang, and F. M. Peeters, Appl. Phys. Lett. 86, 192107 (2005).

${ }^{18}$ E. A. de Andrada e Silva, G. C. La Rocca, and F. Bassani, Phys. Rev. B 50, 8523 (1994).

${ }^{19}$ Y. S. Gui, J. Liu, K. Ortner, V. Daumer, C. R. Becker, H. Buhmann, and L. W. Molenkamp, Appl. Phys. Lett. 79, 1321 (2001).

${ }^{20}$ W. Yang and Kai Chang, Phys. Rev. B 73, 045303 (2006). 